\title{
The South African Arrhythmogenic Right Ventricular Cardiomyopathy Registry: A brief review and a status report
}

\section{Neil Hendricks, Bongani M. Mayosi and Andrzej Okreglicki}

Cardiac Clinic, Groote Schuur Hospital, Department of Medicine, University of Cape Town, South Africa

Address for correspondence:

clo A. Okreglicki

E25 Cardiac Clinic

Groote Schuur Hospital

Observatory

7925

South Africa

Email:

a.okreglicki@uct.ac.za

arvc.sa@uct.ac.za

\section{ABSTRACT Arrhythmogenic Right Ventricular Car-} diomyopathy (ARVC), commonly an inherited condition affecting cell junctions, may present with sudden death or life-threatening arrhythmias. The pathogenesis, clinical presentation, diagnostic evaluation and treatment are reviewed. The South African ARVC Registry is described, giving the aims, organization and missions which include: establishment of a DNA/tissue bank, epidemiology, risk assessment and evaluation of therapy, imaging, pathological diagnosis and diagnostic validation. Results of analysis of the first $\mathbf{8 0}$ confirmed cases enrolled in the registry confirm its familial occurrence, high mortality and involvement of the left ventricle. A common finding is participation in sport. A new mutation has been discovered affecting the plakophyllin gene. Physicians are invited to refer patients with confirmed or suspected ARVC for inclusion in the Registry. SAHeart 2008; 5: 148-154

\section{BACKGROUND}

Arrhythmogenic Right Ventricular Cardiomyopathy (ARVC) is a progressive disorder of the right ventricular myocardium, associated with ventricular arrhythmias, sudden death and heart failure. Despite estimates of 1:2 500 to 1:5000, its true prevalence is unknown, as some affected individuals may remain asymptomatic and others may be misdiagnosed.The importance of this unusual heart muscle disease is in its role as a major cause of sudden cardiac death in the young, especially athletes. ${ }^{(1,2,3,19)}$

\section{PATHOGENESIS}

ARVC is familial in up to $50 \%$ of cases, with an autosomal dominant pattern of inheritance occurring in the majority. Rarer recessive modes of inheritance, however, do occur. One of these may be associated with abnormalities of skin (palmoplantar hyperkeratosis) and hair (wooly hair): the so-called Naxos disease. . $^{(4,6,19)}$

The causative genes encode proteins of cell junctions (plakoglobin plakophilin, desmoglein, desmocollin, desmoplakin), which mediate cellcell interaction, allowing for both mechanical and electrical coupling of adjacent cells. Mechanical coupling is important for providing structural integrity and allowing synchronous contraction of myocardium, while electrical coupling, via gap junctions, allows for rapid spread of depolarizing electrical waves. ${ }^{(4,6,19)}$

Mutations may lead to remodeling of the intercalated disks of cell junctions, resulting in loss of the structural integrity of the myocardium and myocyte death, with replacement by fibrosis and adipocytes. This dystrophy of the right ventricular myocardium with fibro-fatty replacement, which is the pathological hallmark of ARVC serves as substrate for the development of life-threatening arrhythmias (Figure I). ${ }^{(4,6,19)}$

Since heritable penetrance of ARVC is incomplete and genetic mutations within families have been shown to display variable phenotypic expression, both environmental factors and other genetic modifiers have been implicated in its pathogenesis. Endurance sport and intense or prolonged physical activity have been consistently shown in both humans and animal models of ARVC, to be a major modifier in terms of frequency and severity of symptoms, as well an increased risk of death. Endurance sport in ARVC is associated with a 5-fold increase in the risk of sudden death in the young. ${ }^{(6,16,19)}$ 


\section{CLINICAL}

The clinical picture is often described in four "classical" phases: ${ }^{(5)}$

I. The concealed phase, with either subtle or no structural changes, occurs in affected individuals, who despite being asymptomatic, remain at risk of sudden cardiac death (SCD) from an arrhythmia during episodes of extreme exertion. These individuals may be identified through family screening; however, in a significant proportion the diagnosis is first established at postmortem examination, the initial presentation having been SCD.

2. The overt electrical phase is characterized by effort-induced palpitations and syncope. The symptoms are due to ventricular arrhythmias of right ventricular (RV) origin, manifesting as multiple ventricular ectopic beats, sustained and non-sustained ventricular tachycardia (VT) with left bundle branch block (LBBB) morphology. Morphological and functional cardiac abnormalities are usually present at this stage, but often overlooked.

3. Diffuse right ventricular dysfunction occurs as a result of progressive loss of RV myocardium presenting with severe right-sided heart failure, with relatively preserved left ventricular function.

\section{Biventricular failure or dilated cardiomyopathy phase in which both} ventricles are equally affected; ARVC mimicking dilated cardiomyopathy. This may be severe enough to require cardiac transplantation.

\section{DIAGNOSTIC EVALUATION}

A clinical diagnosis is achieved by demonstrating functional and structural alterations of the right ventricle, coupled with depolarization and repolarization abnormalities, arrhythmias with LBBB morphology (Figure 2) and fibro-fatty replacement on histology (Figure I). ARVC is confirmed by a combination of 2 major, I major and 2 minor, or 4 minor criteria (Table I).(4) The main differential diagnoses include idiopathic right ventricular outflow tract tachycardia, myocarditis, dilated cardiomyopathy and sarcoidosis. ${ }^{(4,5,19)}$

An ECG is essential in the evaluation of ARVC, providing both diagnostic and prognostic information. Abnormalities on ECG are detected in up to $90 \%$ of ARVC cases. Features include the presence of diffuse precordial T-wave inversions (beyond V2), an Epsilon wave in VI, widened localized QRS complex in leads VI-V3 (parietal block) and prolonged S-wave upstroke ( $\geq 55 \mathrm{~ms}$ ) in VI-V3 (Figure 3). ${ }^{(8,9,19}$

T-wave inversion in leads VI-V3 in the absence of RBBB and coronary disease is a well-established electrocardiographic feature of ARVC and is a minor criterion (Table I). An epsilon wave (defined as a discrete wave following the QRS complex in VI-V3) and parietal block are both major diagnostic criteria (Table I). Late action potentials on signal averaged electrocardiogram (SAECG) recordings are the counterpart of the epsilon waves, demonstrating the presence of areas of slow conduction and late depolarization, and predict inducibility of ventricular tachycardia (VT) on electrophysiological study (EPS), as well as implantable cardioverter defibrillator (ICD) therapies. ${ }^{(17,19)}$ Prolonged

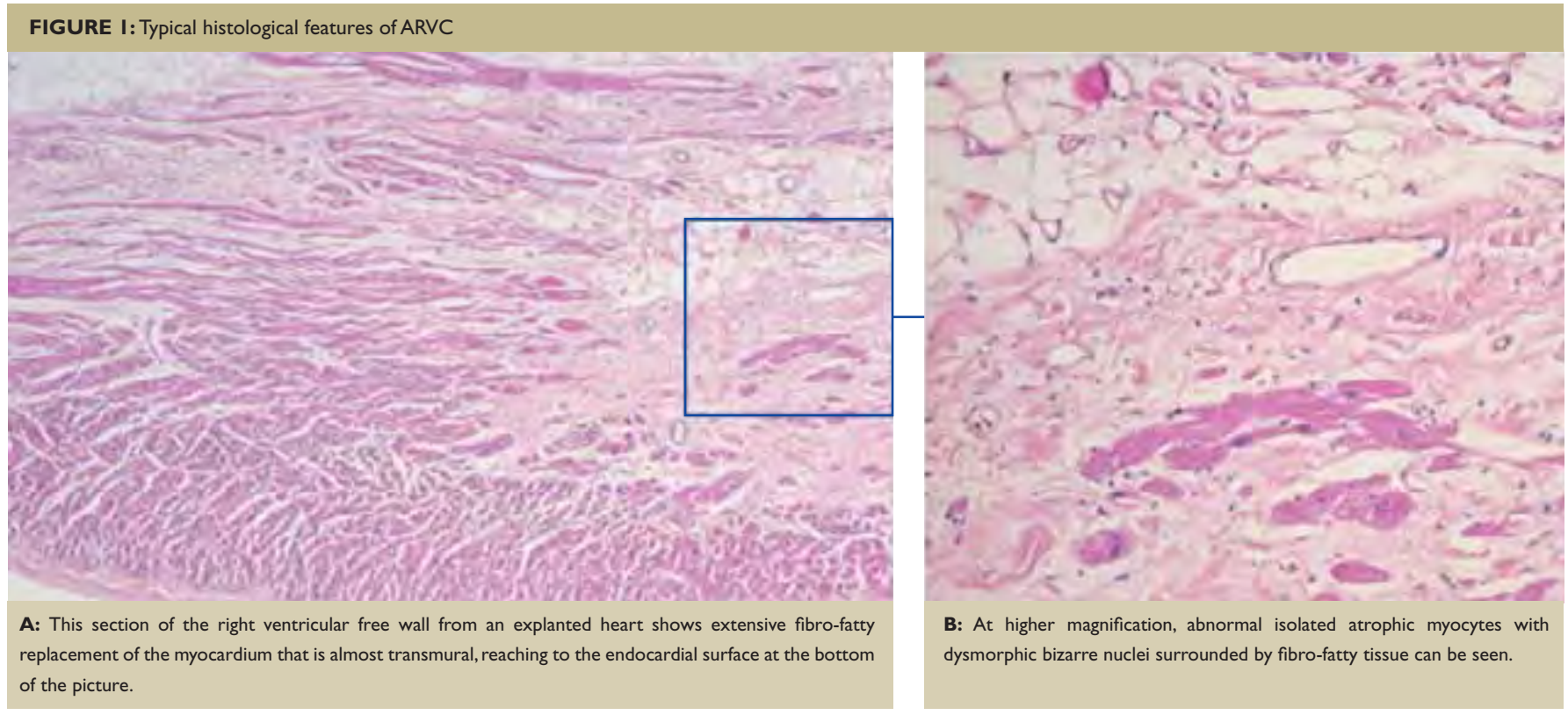


TABLE I: Diagnostic Criteria for ARVC

\begin{tabular}{|c|c|c|}
\hline Category & Major Criteria & Minor Criteria \\
\hline $\begin{array}{l}\text { Structural or Functional } \\
\text { abnormalities }\end{array}$ & $\begin{array}{l}\text { 1. Severe dilatation and } \\
\text { reduction of RV ejection } \\
\text { fraction with mild or no } \\
\text { LV involvement. } \\
\text { 2. Localized RV aneurysms } \\
\text { (akinetic or dyskinetic } \\
\text { areas with diastolic } \\
\text { bulgings). } \\
\text { 3. Severe segmental } \\
\text { dilatation of RV. }\end{array}$ & $\begin{array}{l}\text { I. Mild global RV dilatation } \\
\text { and/or ejection fraction } \\
\text { reduction with normal LV. } \\
\text { 2. Mild segmental dilatation } \\
\text { of RV. } \\
\text { 3. Regional RV hypokinesia. }\end{array}$ \\
\hline Tissue Characterization & $\begin{array}{l}\text { Fibro-fatty replacement of } \\
\text { myocardium on histology }\end{array}$ & \\
\hline $\begin{array}{l}\text { ECG Depolarization / } \\
\text { Conduction abnormalities }\end{array}$ & $\begin{array}{l}\text { 1. Epsilon waves or } \\
\text { 2. Localized prolongation } \\
\text { (>110 ms) of QRS } \\
\text { complex in right } \\
\text { precordial leads (VI-V3). }\end{array}$ & Late potentials on SAECG \\
\hline $\begin{array}{l}\text { ECG Repolarization } \\
\text { abnormalities }\end{array}$ & & $\begin{array}{l}\text { Inverted T waves in VI-V3 } \\
\text { (above age I } 2 \text { yrs, in } \\
\text { absence of RBBB) }\end{array}$ \\
\hline Arrhythmias & & $\begin{array}{l}\text { I. LBBBVT on ECG or } \\
\text { Holter monitoring or } \\
\text { during exercise testing. } \\
\text { 2. Frequent PVCs } \\
(>1000 / 24 \mathrm{~h} \text { on Holter })\end{array}$ \\
\hline Family History & $\begin{array}{l}\text { Familial disease confirmed } \\
\text { at autopsy or biopsy. }\end{array}$ & $\begin{array}{l}\text { I. Family history of } \\
\text { premature sudden death } \\
\text { (<35 years of age) due } \\
\text { to suspected ARVC. } \\
\text { 2. Family history (clinical } \\
\text { diagnosis based on } \\
\text { present criteria) }\end{array}$ \\
\hline
\end{tabular}

Abbreviations: $R V=$ right ventricle, $L V=$ left ventricle, $A R V D=$ arrhythmogenic right ventricular dysplasia, $\mathrm{RBBB}=$ right bundle branch block, $\mathrm{LBBB}=$ left bundle branch, $\mathrm{VT}=$ ventricular tachycardia, PVC = premature ventricular complex (Modified from McKenna et al.) $)^{(4)}$
S-wave upstroke in $\mathrm{VI-V3}$, the most prevalent electrocardiographic feature, is seen in $95 \%$ of the patients. It correlates with disease severity and the induction of ventricular tachycardia at EPS. (9,19)

EPS should be considered in all individuals where ARVC is suspected or confirmed, as it has important diagnostic, prognostic and therapeutic utility. Inducibility of VT during EPS, correlates with prognosis,

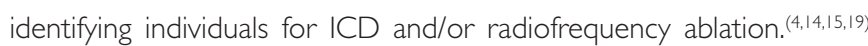
Electroanatomic voltage mapping is able to detect areas of low voltage corresponding to myocardial atrophy with fibro-fatty replacement, enabling distinction between ARVC and idiopathic right ventricular outflow tract tachycardia. As the disease process is patchy, this mapping also allows for more directed targeting of endomyocardial biopsy.(18,19)

RV angiography (RVA) is the imaging gold standard in ARVC and may demonstrate the classical features of localized aneurysms, prominent trabeculations of the RV and global dilation with decreased ejection fraction (Figure 4). . $^{(9,21)}$

Echocardiography, despite being widely available and frequently performed, has a low sensitivity for the detection of ARVC due to the difficulty of imaging the RV. Despite these shortcomings, it remains useful as the first-line non-invasive imaging modality in assessing both presence of disease at primary evaluation in suspected ARVC and in 


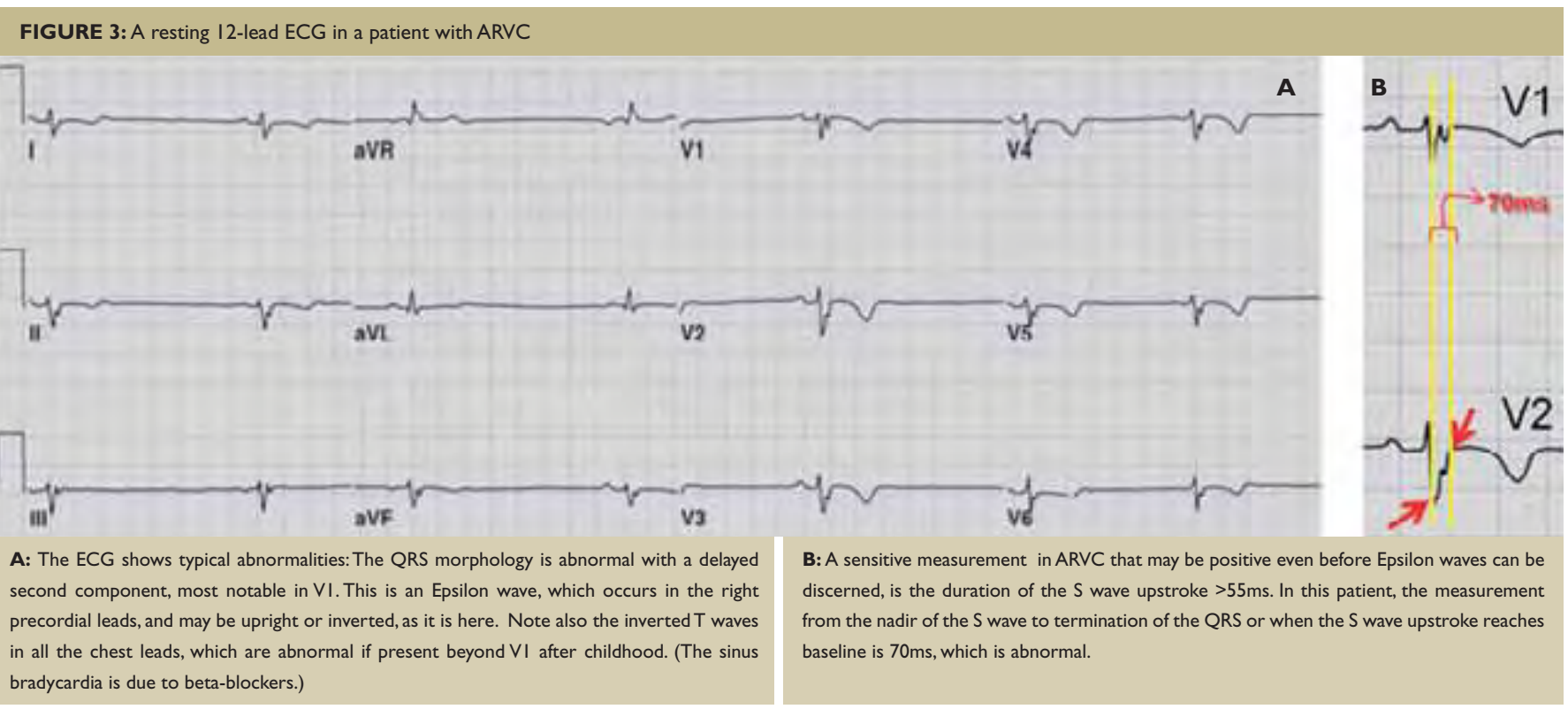

family screening and also, by means of serial examinations, progression during the follow-up of affected patients. Both functional and structural abnormalities can be evaluated, the detection of which may avoid the need for invasive RVA or costly cardiac magnetic resonance imaging $(\mathrm{CMRI})^{(10,19)}$

CMRI has become integral to the evaluation of ARVC because it is noninvasive, with increased sensitivity over echocardiography. Accurate measurements of both RV and LV size, volume and ejection fraction are obtained. Wall motion abnormalities with akinesia, dyskinesia and aneurysms diagnostic of ARVC are readily appreciated, as well as

TABLE 2: Points to Remember about ARVC

\section{Symptoms}

Fainting episodes, especially with exercise

Episodes of transient or persistent palpitations; may be associated with lightheadedness

\section{Diagnostic Clues}

\section{Family History}

Unexplained sudden death in young or middle aged family member(s)

\section{ECG findings}

T-wave inversions beyond lead $\mathrm{VI}$

Epsilon waves in leads VI-V3

Ventricular premature beats with left bundle branch configuration

Abnormal electrical potentials in high-resolution (signal-averaged) ECG

\section{Echocardiography}

Dilated RV with aneurysms

Treatment

Antiarrhythmic medications

ICD

Radiofrequency ablation

Heart transplant evidence of fibrosis and fatty infiltration (Figure 5). However, the contraindication of CMRI in the presence of ICDs, high inter-observer variability coupled with a lack of standardized protocols producing variable results have resulted in CMRI disappointingly not living up to its initial expectations as the definitive mode of investigation. This is of particular importance in the South African context with its lack of expertise in the field of CMRI. In the case of the diagnosis of ARVC, it

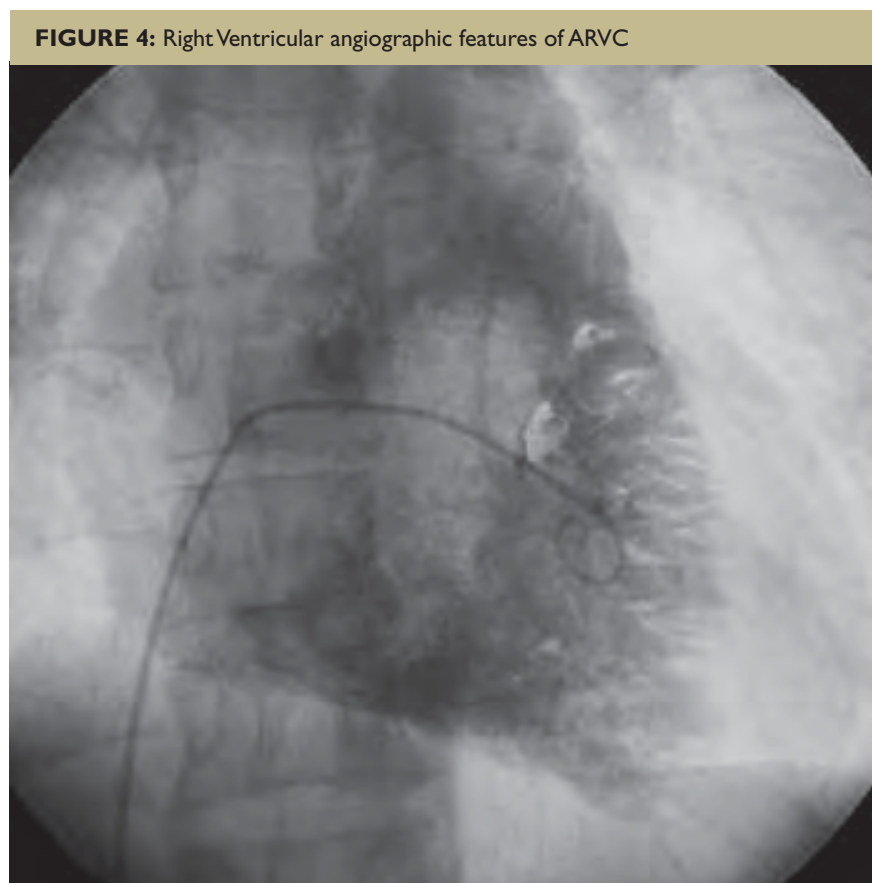

This RV angiogram would be best appreciated in "movie" mode as it shows hypokinesia of the entire anterior wall and apex. Other typical abnormalities can be noted here: along the anterior wall prominent trabeculations, which may have a "stack of coins" appearance, and localized aneurysms closer to the RV outflow region. 


\section{THE SA ARVC REGISTRY}

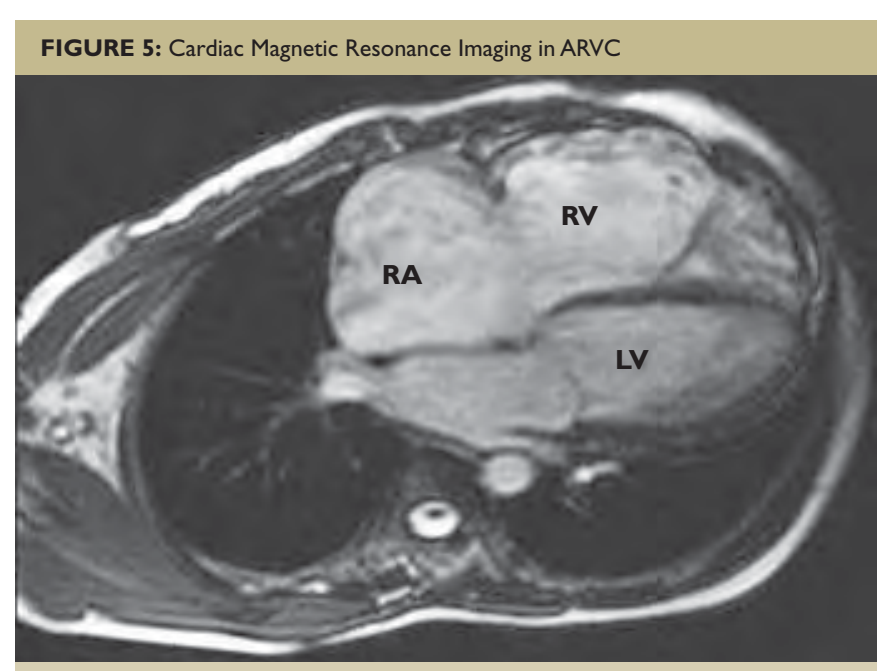

This MRI 4-chamber view of a patient with advanced ARVC shows some of the typical features: dilated right ventricle (RV), dilated right atrium (RA), multiple aneurysms of the RV free wall. The left ventricle (LV) is displaced posteriorly, in this patient, by the massive RV. The LV size is preserved.

has been shown that the sensitivity and specificity of CMRI are directly related to the experience of the radiologist in the reading of ARVC cases. $^{(11,12,19)}$ Multidetector Cardiac CT has shown promise as an alternative imaging modality with the added benefit of coronary anatomy evaluation. (13) $^{1}$

\section{TREATMENT}

Treatment of ARVC is directed toward preventing life-threatening ventricular arrhythmias. This is achieved by sports avoidance and a combination of anti-arrhythmic drugs, radiofrequency ablation and ICDs. ${ }^{(5,14,15,19)}$

All forms of intense physical activity and endurance sport should be prohibited; competitive sport is also to be avoided. These activities are associated with an unacceptably high risk of death and avoidance has been shown to be associated with a reduction in SCD among participants. An exercise test may be of benefit in identifying individuals at risk for developing exercise-induced arrhythmias but, as it is unable to mimic the competitive sport situation with its probable associated catecholamine rise and sympathetic effects, its sensitivity is low. ${ }^{(16,22)}$

Beta-blockers are the most commonly used drugs. Alternative agents include sotalol and amiodarone.

Radiofrequency ablation (RFA) may be used as the sole treatment modality in selected cases where the $\mathrm{VT}$ originates from a focal area amenable to ablation; however, it is mostly combined with anti- arrhythmic agents or an ICD. It is indicated particularly for drug failure and break-through arrhythmias or frequent ICD shock therapy. Although effective in the short term, RFA is not curative and is associated with a high recurrence of $\mathrm{VT}$ of $40 \%$ at 3 years, probably due to the progressive nature of the disease. ${ }^{(14)}$

Major risk factors for adverse prognosis are: young age, family history of juvenile sudden death, QRS dispersion $\geq 40 \mathrm{~ms}$, T-wave inversion left ventricular involvement, cardiac failure, ventricular tachycardia, syncope and previous cardiac arrest. Sports avoidance, ICDs and heart transplant (in severe heart failure) are life-saving interventions. ${ }^{(14,15,16,19}$

\section{SA ARVC REGISTRY}

International registries have been established in order to address issues relating to pathogenesis, prognosis and treatment. Much of our understanding of ARVC emanates from this. ${ }^{(5,6,7,9,11,12,14,19,20)}$ To this end the SA ARVC registry, under the steering of the Working Group on Registries of the Cardiac Arrhythmia Society of Southern Africa (CASSA) was established. The coordinating centre is based in the Cardiac Clinic, Groote Schuur Hospital, Cape Town. Ethics approval had been obtained from the Research Ethics Committee of the University of Cape Town Faculty of Health Sciences.

Prospective enrolment has taken place since January 2004. Physicians refer suspected cases to the registry coordinator. After the diagnosis has been confirmed by the diagnostic panel, patients are invited, with consent, to partake in the registry. Written informed consent is obtained for blood samples for DNA analysis and for release to the registry and storage of any biopsy material obtained previously during diagnostic work-up by the treating physician. Additional written consent is obtained for any further investigations which may be performed to confirm the diagnosis of ARVC, to determine the extent or severity of the disease or for prognostication purposes.

The functional organization of the registry has been formalized (see the organogram in Figure 6). The registry has six major missions: DNA/ Tissue bank, epidemiology, risk assessment and evaluation of therapy, imaging, pathological diagnosis and diagnostic validation.

\section{DNA / Tissue banking}

Blood samples for DNA analysis are obtained from all confirmed cases and family members to determine gene loci and to identify specific abnormal genes. 
This will enable genotype-phenotype correlation and facilitate family screening for identified mutations.

\section{Epidemiology}

The screening for and the detection of familial occurrence by means of ECG, SAECG, Holter 24-hour ECG recording, exercise stress test, echocardiography, CMRI and genetic screening of known mutations will expand the limited epidemiological database of this condition in southern Africa. The pathology reports of explanted hearts obtained at cardiac transplantation, will be reviewed for features consistent with ARVC. Anatomical and forensic pathologists across South Africa will be encouraged to forward identified potential cases for review.

\section{Risk stratification and evaluation of therapies}

The main aim is to identify prognostic factors and evaluate long-term efficacy of current pharmacological and non-drug therapy.

\section{Imaging}

In addition to evaluating the diagnostic and prognostic value of existing imaging modalities and techniques, new MRI protocols, such as those utilizing cine Displacement Encoding with Stimulated Echoes (cine DENSE) for quantifying regional myocardial strain based on phase contrast images optimized for RV, are being developed using the registry patients to enhance earlier disease detection.

\section{Pathology registry and tissue bank}

A cardiac pathology registry has been set up to collect either heart specimens or endomyocardial biopsies. Protocols have been developed for processing and analysis of these specimens including immunohistochemistry, histochemistry, electron microscopy and myocyte culture. Insight will be gained into the etiopathogenesis of the disease and the diagnostic value of endomyocardial biopsy in the workup of suspected cases of ARVC.

\section{RESULTS AND REGISTRY DATA}

To date, 80 confirmed cases of ARVC, 70 being probands, have been enrolled in the registry of whom 54/80 (68\%) were males. The median age at diagnosis was 27.5 years (range II-74 years), 23/80 (29\%) of whom were 20 years or younger. ARVC has been diagnosed in all racial groups.

With regard to inheritance, 48/80 (60\%) had either a family member with confirmed ARVC or a history of SCD or sudden unexplained death. LV involvement in ARVC, a measure of the extent of the disease, was present in 35/80 (44\%), 4 of whom subsequently had heart transplants with another awaiting transplantation. Other relevant therapy data reveal that ICDs were implanted in 30/80 (38\%).

Assessment of potential risk factors shows that 40/80 (50\%) participated in some form of organized sport; 4/80 (5\%) were survivors of SCD which occurred whilst participating in sporting activity.

FIGURE 6: The Organogram of the SA ARVC Registry

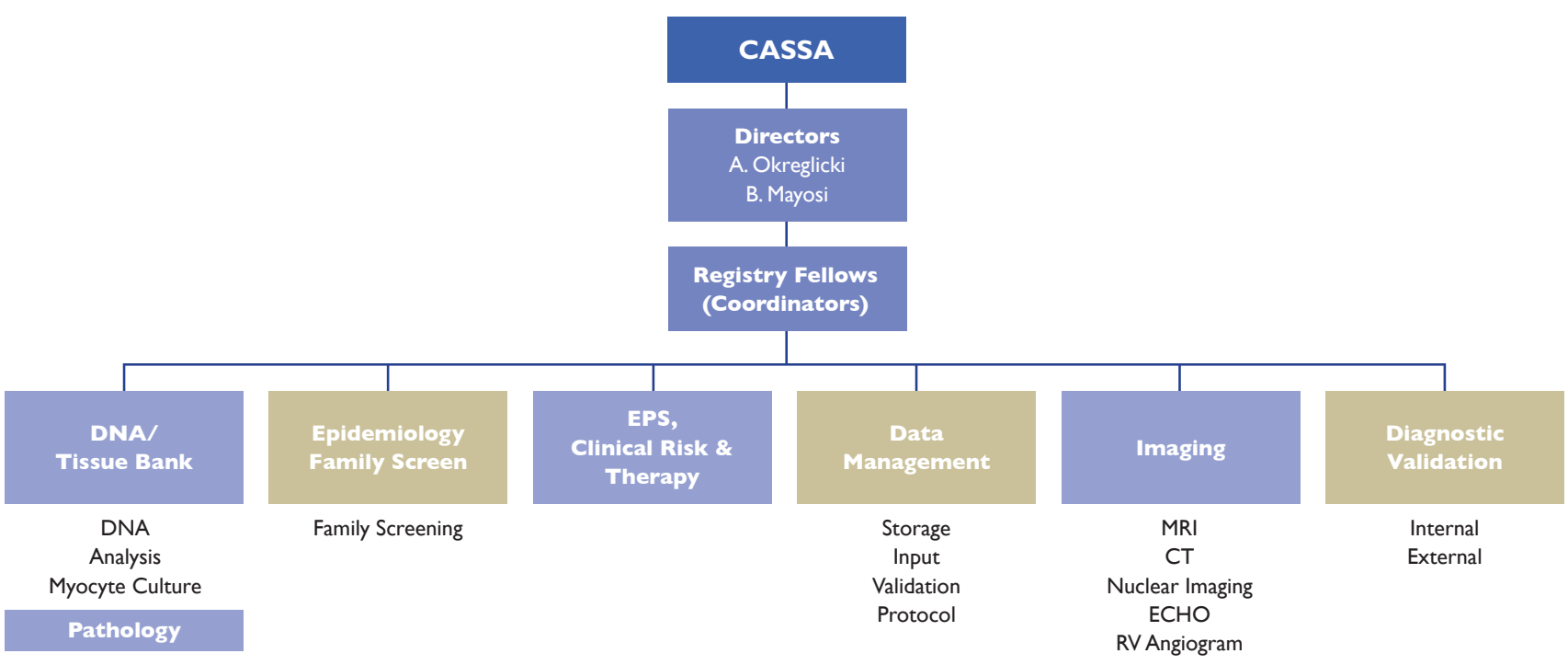




\section{THE SA ARVC REGISTRY}

Follow-up data are available for 66/80 (83\%) of whom I4/66 (21\%) have died.

A novel genetic mutation in the plakophyllin gene was discovered in one family that presented with early progressive disease proceeding to cardiac transplantation in 2 siblings.

\section{DISCUSSION}

This registry represents the largest cohort of ARVC patients from the African continent. The results confirm the clustering of ARVC in families, the significant role of endurance sport and the high mortality of $\operatorname{ARVC}\left({ }^{(4,6,16,19)}\right.$

Despite the short existence of the registry and minimal financia support, considerable strides have been made with respect to recruitment, data collection and novel findings. In this regard it compares favourably with the output of other international registries. ${ }^{(20)}$

There remains a call for cardiologists and physicians across South Africa to be vigilant in identifying affected individuals and to be diligent in referring all suspected or confirmed cases of ARVC to the registry in order for it to achieve its goals.

Contact details for referral of confirmed and suspected cases of ARVC arvc.sa@uct.ac.za

\section{REFERENCES:}

I. Thiene G, Nava A, Corrado D, et al. Right ventricular cardiomyopathy and sudden death in young people. N Engl J Med 1988;318:129-33.

2. Nava A, Rossi L, Thiene G, editors. Arrhythmogenic right ventricular cardiomyopathy/ dysplasia. Elsevier, Amsterdam; 1997.

3. Basso C, Thiene G, Corrado D, et al. Arrhythmogenic right ventricular cardiomyopathy. Dysplasia, dystrophy, or myocarditis? Circulation 1996;94:983-91.

4. McKenna WJ, Thiene G, Nava A, et al. Diagnosis of arrhythmogenic right ventricular dysplasia/cardiomyopathy. Br Heart J 1994;71:215-8.

5. Sen-Chowdhry S, Lowe MD, Sporton S, McKenna W. Arrhythmogenic right ventricular cardiomyopathy: clinical presentation, diagnosis and management. Am J Med 2004; 1 17:685-695.

6. Awad M, Calkins H, Judge D. Mechanisms of Disease: molecular genetics of arrhythmogenic right ventricular dysplasia/cardiomyopathy. Nature Clinical Practice Cardiovascular Medicine 2008;5:258-267.

7. Calkins H. Progress Continues in Our Understanding of the Electrocardiographic Manifestations of Arrhythmogenic Right Ventricular Dysplasia. Journal of Cardiovascular Electrophysiology 2008; 19:782-783

8. Cox M, Nelen M, Wilde A, et al. Activation delay and $V T$ parameters in arrhythmogenic right ventricular dysplasia/cardiomyopathy: Towards improvement of diagnostic ECG criteria. J Cardiovasc Electrophysiol 2008; 19:775-81.

9. Nasir K, Bomma C,Tandri H, et al. Electrocardiographic features of arrhythmogenic right ventricular dysplasia/cardiomyopathy according to disease severity: a need to broaden diagnostic criteria. Circulation 2004; I 10:1527- 534.

10. Scheinman M, Crawford M. Echocardiographic findings and the search for a gold standard in patients with Arrhythmogenic Right Ventricular Dysplasia. JACC 2005; 45:866-7.

II. Tandri $H$, Macedo $R$, Calkins $H$, et al. Role of magnetic resonance imaging in arrhythmogenic right ventricular dysplasia: insights from the North American arrhythmogenic right ventricular dysplasia (ARVD/C) study. Am Heart J 2008; |55:| 47-53

12. Sen-Chowdhry S, McKenna WJ. The utility of magnetic resonance imaging in the evaluation of arrhythmogenic right ventricular cardiomyopathy. Current Opinion in Cardiology 2008;23:38-45

13. Plumhans C, Muhlenbruch G, Rapaee A, et al. Assessment of global right ventricular function on 64-MDCT compared with MRI. AJR 2008; | 90: | 358- | 36 |.

14. Wichter T, Paul M, Eckardt L, et al. Arrhythmogenic right ventricular cardiomyopathy Antiarrhythmic drugs, catheter ablation, or ICD? Herz 2005;30:91- I01.

I5. Wichter T, Paul M, Wollmann C, et al. Implantable cardioverter/defibrillator therapy in arrhythmogenic right ventricular cardiomyopathy: single-center experience of long-term follow-up and complications in 60 patients. Circulation 2004; 109:1 503-8.

16. Corrado D, Basso C, Schiavon M, Thiene G. Does sports activity enhance the risk of sudden death in adolescents and young adults? J Am Coll Cardiol 2003;42:1959- 1963.

17. Nasir K, Tandri H, Rutberg J, et al. Filtered QRS duration on signal-averaged electrocardiography predicts inducibility of ventricular tachycardia in arrhythmogenic right ventricle dysplasia. Pacing Clin Electrophysiol 2003;26:1955-1960.

18. Corrado D, Basso C, Leoni L, et al.Three-dimensional electroanatomic voltage mapping increases accuracy of diagnosing arrhythmogenic right ventricular cardiomyopathy/ dysplasia. Circulation. 2005; 1 । 1:3042-50.

19. Thiene G, Corrado D, Basso C. Arrhythmogenic right ventricular cardiomyopathy/ dysplasia. Orphanet Journal of Rare Diseases 2007;2:45

20. Basso C, Wichter T, Gian A, et al. Arrhythmogenic right ventricular cardiomyopathy: clinical registry and database, evaluation of therapies, pathology registry, DNA banking. European Heart Journal 2004;25:531-534.

21. Daliento L, Rizzoli G, Thiene G, et al. Diagnostic accuracy of right ventriculography in arrhythmogenic right ventricular cardiomyopathy. Am J Cardiol 1990;66:74I-745.

22. Giada F, Barold S, Biffi A, et al. Sport and arrhythmias: summary of an international symposium. European Journal of Cardiovascular Prevention and Rehabilitation 2007 $|4: 707-7| 4$ 